\title{
USO DE ANTIMICROBIANOS POR GESTANTES NO SERVIÇO PÚBLICO DE SAÚDE
}

\author{
Use of antimicrobials by pregnant women in the public health \\ care
}

\author{
Uso de antimicrobianos por embarazadas del servicio público \\ de salud
}

Artigo Original

\section{RESUMO}

Objetivo: Identificar e classificar quanto ao risco os antimicrobianos prescritos para gestantes atendidas no Programa Nacional de Acompanhamento Pré-Natal e Puerpério em um município do Rio Grande do Sul. Métodos: Estudo transversal analítico e retrospectivo, realizado em 2011, a partir do cadastro de 87 gestantes atendidas em 2010, por meio de um instrumento com questões relacionadas aos medicamentos dispensados. Os antimicrobianos prescritos foram identificados e classificados como de uso tópico e sistêmico, e quanto à categoria de risco de uso na gravidez, com tratamento realizado pelo SPSS 15.0. Resultados: As gestantes tinham idade média de 28,01 anos, realizaram 5,3 consultas. Foram prescritos em média 6,52 medicamentos/gestante, dos 568 medicamentos prescritos, 85 (14,96\%) com ação antimicrobiana, $29(34,1 \%)$ de uso tópico e $56(65,9 \%)$ uso sistêmico, sendo que 46 $(52,9 \%)$ gestantes receberam pelo menos um antimicrobiano. Encontrou-se 13 produtos distintos, prevalecendo antifúngicos de uso ginecológico/tópico, 16 (18,82\%), 30 (35,3\%) foram prescritos no primeiro trimestre de gravidez, $30(35,3 \% \%)$ no segundo e $25(29,4 \%)$ no terceiro. Conforme a classificação de risco ao feto, nove $(61,54 \%)$ antimicrobianos são da categoria B e quatro $(30,77 \%)$ da categoria C. Conclusão: Verificou-se a prescrição de antimicrobianos de uso sistêmico e de risco B na gestação para 52,9\% das gestantes em acompanhamento pré-natal no local do estudo. Miconazol e metronidazol creme vaginal, cefalexina $500 \mathrm{mg}$, azitromicina $500 \mathrm{mg}$ e amoxicilina $500 \mathrm{mg}$ foram os mais prescritos.

Descritores: Antibacterianos; Uso de medicamentos; Gravidez.

\section{ABSTRACT}

Objective: To identify and classify, according to the risk, the antimicrobials prescribed to pregnant women assisted by the National Program of Prenatal and Postpartum Follow-up in a municipality of Rio Grande do Sul. Methods: Analytical and retrospective cross-sectional study conducted in 2011 based on the registers of 87 pregnant women assisted in 2010 using an instrument with questions about the dispensed drugs. The prescribed antimicrobials were identified and classified as topical and systemic use and according to the risk of using it during pregnancy. Data were analyzed using SPSS 15.0. Results: The pregnant women had a mean age of 28.01 years and attended 5.3 consultations. There was an average prescription of 6.52 drugs/pregnant woman. Of the 568 drugs prescribed, 85 (14.96\%) had an antimicrobial activity, 29 (34.1\%) were for topical use and 56 (65.9\%) for systemic use, and 46 (52.9\%) pregnant women received at least one antimicrobial. There were 13 different products with a prevalence of antifungals of gynecological / topical use, 16 (18.82\%), 30 (35.3\%) were prescribed in the first trimester of pregnancy, 30 (35.3\%) in the second and $25(29.4 \%)$ in the third trimester. According to the classification of the risk to the fetus, nine $(61.54 \%)$ antimicrobials belonged to the B category and four (30.77\%) to the C category. Conclusion: It was verified the prescription of antimicrobials for systemic use and B category risk for pregnancy to $52.9 \%$ of pregnant women in prenatal follow-up in the place where the study took place. Miconazole and metronidazole vaginal cream, cephalexin $500 \mathrm{mg}$, azithromycin $500 \mathrm{mg}$ and amoxicillin $500 \mathrm{mg}$ were the most prescribed drugs.

Descriptors: Anti-bacterial Agents; Drug Utilization; Pregnancy.

Recebido em: 27/04/2013 Revisado em: 29/01/2014 Aceito em: 10/03/2014 


\section{RESUMEN}

Objetivo: Identificar y clasificar el riesgo de los antimicrobianos prescritos a las embarazadas asistidas en el Programa Nacional de Atención Prenatal y Puerperio en el municipio de Rio Grande do Sul. Métodos: Estudio transversal analítico y retrospectivo realizado en 2011 con el registro de 87 embarazadas asistidas en 2010 a través de un instrumento con preguntas relacionadas a los medicamentos dispensados. Los antimicrobianos prescritos fueron identificados y clasificados de uso tópico y sistémico y respecto la categoría de riesgo del uso en el embarazo, con análisis realizado por el SPSS 15.0. Resultados: Las embarazadas tenian edad media de 28,01 años y realizaron 5,3 consultas. Fueron prescritos una media de 6,52 medicamentos/embarazada, de los 568 medicamentos prescritos, 85 (14,96\%) con acción antimicrobiana, $29(34,1 \%)$ de uso tópico y 56 (65,9\%) de uso sistémico, siendo que $46(52,9 \%)$ de las embarazadas recibieron por lo menos un antimicrobiano. Se encontró 13 productos distintos con prevalencia de anti fúngicos de uso ginecológico/ tópico, 16 (18,82\%), 30 (35,3\%) fueron prescritos en el primer trimestre del embarazo, $30(35,3 \% \%)$ en el segundo y 25 (29,4\%) en el tercero. Según la clasificación de riesgo al feto, nueve (61,54\%) antimicrobianos son de la categoría B y cuatro $(30,77 \%)$ de la categoría $C$. Conclusión: Se verificó la prescripción de antimicrobianos de uso sistémico y de riesgo $B$ en el embarazo para el 52,9\% de las embarazadas en atención prenatal en el sitio del estudio. Miconazol y metronidazol crema vaginal, cefalexina $500 \mathrm{mg}$, azitromicina $500 \mathrm{mg}$ y amoxicilina $500 \mathrm{mg}$ fueron los más prescritos.

Descriptores: Antibacterianos; Utilización de Medicamentos; Embarazo.

\section{INTRODUÇÃO}

A utilização de medicamentos por gestantes e as consequências sobre as crianças que vão nascer passou a ser objeto de grande preocupação após fatos ocorridos entre o final da década de 50 e início dos anos $60^{(1)}$.

A gestação, por suas peculiaridades biológicas, torna a mulher e seu concepto particularmente expostos a riscos, entre os quais se destacam os relacionados ao consumo de medicamentos $^{(2)}$. A resposta do feto ao uso de medicamentos é diferente da observada na mãe, podendo resultar em toxicidade fetal, com lesões variadas, sendo que algumas podem ser irreversíveis ${ }^{(3)}$.

O uso de fármacos na gestação deve ser evitado, no entanto, em algumas situações, esse uso é indispensável ${ }^{(4)}$, podendo se tornar danoso para mulheres portadoras de doenças crônicas ou que sofram intercorrências médicas durante a gestação ${ }^{(5)}$. As informações sobre os riscos do uso de medicamentos durante a gestação são bastante limitadas, tanto em quantidade como em qualidade, o que se deve fundamentalmente às dificuldades éticas e metodológicas da pesquisa nessa área ${ }^{(4)}$.

As informações sobre o risco da utilização de medicamentos durante a gravidez são escassas, o uso indiscriminado decorrente de automedicação ou de prescrições médicas sem critérios técnico-científicos é relatado em diferentes países ${ }^{(1)}$. Estudo realizado com mulheres em gestação de alto risco verificaram associações estatisticamente significativas entre automedicação em mulheres com menor número de consultas de pré-natal, uso de cigarro na gestação e maior número de filhos ${ }^{(6)}$.

Tendo em vista que, durante a gestação, infecções do trato urinário (ITU) são bastante frequentes, sendo que até $10 \%$ das gestantes podem apresentar algum episódio de infecção urinária, a maioria assintomática ${ }^{(4)}$, justifica-se a prescrição de fármacos antimicrobianos neste período. Em estudo $^{(7)}$ que analisou prescrições emitidas para gestantes internadas em Hospital do município de Mirassol/ $\mathrm{SP}$, os antimicrobianos estiveram presente em $40 \%$ das prescrições, sendo que os mais prescritos foram cefalotina $(10,8 \%)$ e cefalexina $(4,6 \%)$. Outro estudo ${ }^{(8)}$ com gestantes que se apresentaram para consulta em serviço de prénatal do Sistema Único de Saúde (SUS) em seis cidades brasileiras, os antimicrobianos foram utilizados por $11,1 \%$ das gestantes e corresponderam a $6,9 \%$ dos medicamentos utilizados.

Além da incidência aumentada da ITU e de outras infecções entre grávidas, neste período o arsenal terapêutico antimicrobiano e as possibilidades profiláticas são restritas, considerando-se a toxicidade dos fármacos para o feto ${ }^{(9)}$.

Neste contexto, destaca-se que grande parte dos medicamentos com ação antimicrobiana, de acordo com a classificação de risco ao feto, pertence à categoria $\mathrm{B}^{(4)}$, indicando que foram realizados estudos em reprodução animal com estes fármacos, os quais não demonstraram risco fetal, entretanto, sem realização de estudos controlados em mulheres grávidas; ou os estudos em reprodução animal demonstraram efeitos adversos que não foram confirmados em estudos controlados em mulheres no primeiro trimestre, mas não há evidência de risco nos demais trimestres ${ }^{(10)}$.

Diante do exposto, o objetivo do presente estudo foi identificar e classificar quanto ao risco os antimicrobianos prescritos para gestantes atendidas no Programa Nacional de Acompanhamento Pré-Natal e Puerpério em um município do Rio Grande do Sul.

\section{MÉTODOS}

Realizou-se estudo transversal e retrospectivo, com prescrições das gestantes atendidas no Programa Nacional de Acompanhamento Pré-Natal e Puerpério em uma 
Unidade Básica de Saúde (UBS - Central) e no Centro de Atendimento aos Adolescentes do Município de Ijuí/RS (CAAMI), no ano de 2010.

A coleta de dados ocorreu com acesso aos cadastros de todas das gestantes atendidas nos locais pesquisados, no início de 2011, com auxílio de um instrumento de coleta de dados que continha questões relacionadas com as características socioeconômicas destas, bem como os medicamentos dispensados para as gestantes nestes locais.

A coleta de dados ocorreu com pesquisadores treinados e as informações obtidas dos dois locais: Unidade Básica de Saúde (UBS - Central) e Centro de Atendimento aos Adolescentes do Município de Ijuí/RS (CAAMI), por meio do acesso ao prontuário das gestantes e do sistema informatizado de dispensação existente nestes locais. Para auxílio na coleta de dados, foi criado um instrumento que era preenchido por uma pesquisadora e posteriormente validado por outro. Em média eram analisados 20 registros por dia.

Incluíram-se no presente estudo todas as gestantes atendidas nos locais supracitados, que receberam a prescrição de pelo menos um medicamento. Excluíramse mulheres não gestantes, gestantes que não receberam prescrição de medicamentos e gestantes atendidas em outras Unidades de Saúde do município.

Os medicamentos antimicrobianos prescritos foram identificados a partir do seu nome genérico e da forma farmacêutica, o que permitiu classificá-los como de uso tópico e sistêmico.

Os antimicrobianos também foram agrupados quanto à categoria de risco de uso na gravidez, conforme a Food and Drug Administration (FDA), descrita a seguir: categoria A: estudos em mulheres não demonstraram risco ao feto no primeiro trimestre e a possibilidade de prejuízo ao feto parece remota; categoria $\mathrm{B}$ : os estudos em reprodução animal não demonstraram risco fetal, embora não haja estudos em mulheres grávidas, ou os estudos em reprodução animal demonstraram efeito adverso que não foi confirmado em estudos em mulheres no primeiro trimestre; categoria $\mathrm{C}$ : os estudos em animais revelaram efeitos adversos sobre o feto, e não há estudos em mulheres, ou os estudos em mulheres e animais não estão disponíveis. Os fármacos devem ser empregados somente se o benefício potencial justificar o risco potencial ao feto; categoria D: há evidência positiva de risco sobre feto humano, mas os benefícios do uso em mulheres gestantes podem ser aceitáveis a despeito do risco; categoria $\mathrm{X}$ : estudos em animais ou humanos demonstraram anormalidades fetais ou há evidência de risco fetal baseado em experiência humana, ou ambos, e o risco de uso do fármaco em mulheres gestantes excede claramente qualquer possível benefício ${ }^{(11)}$.
A interpretação dos dados ocorreu de acordo com o número de medicamentos total utilizados pelas gestantes, identificação e quantificação dos antimicrobianos, bem como, o período gestacional em que estes foram prescritos, analisando o risco/ benefício dessa utilização durante o período. Realizou-se esta análise de forma descritiva, com média e desvio padrão, sendo realizada a correlação entre o número de medicamentos e o número de consultas médicas por meio da correlação de Spearman, cujo tratamento estatístico foi elaborado com auxílio do software Statistical Package for the Social Sciences (SPSS) (versão 18.0).

A pesquisa recebeu aprovação do Comitê de Ética em Pesquisa da Unijuí sob Parecer Consubstanciado $n^{\circ} 153 / 2011$. Para acesso ao banco de dados foi obtida autorização do local de pesquisa, e os pesquisadores e os responsáveis pelos locais investigados assinaram o termo de sigilo, para garantir a confidencialidade dos dados.

\section{RESULTADOS}

A amostra foi constituída por 87 gestantes que tiveram acompanhamento pré-natal, todas atendidas pelo Sistema Único de Saúde (SUS). A média de idade das gestantes foi de $28,01 \pm 6,61$ anos. Com relação à faixa etária, 41 (47,13\%) encontrava-se com idades entre 20 - 29 anos (Tabela I).

Tabela I - Distribuição das gestantes atendidas no período de estudo conforme a faixa etária atendidas na UBS Central e no CAAMI do município de Ijuí-RS, no ano de 2010. Ijuí/ RS, 2011.

\begin{tabular}{lc}
\hline Faixa Etária & n (\%) \\
\hline $16-19$ & $12(13,79)$ \\
$20-29$ & $41(47,13)$ \\
$30-39$ & $29(33,33)$ \\
$\geq 40$ anos & $5(5,75)$ \\
Total & $87(100)$ \\
\hline
\end{tabular}

A média de consultas realizadas pelas gestantes foi de 5,3 3,01. Na Tabela II pode-se observar o número e a média de medicamentos prescritos às gestantes em relação ao número de consultas pré-natal, contudo, o coeficiente de correlação de Spearman mostrou correlação positiva fraca, significativa $(\mathrm{r}=0,281 ; \mathrm{p}=0,008)$, entre o número de consultas e o número de medicamentos, apesar de não ter associação entre as variáveis.

Encontrou-se a prescrição de 568 medicamentos, em média 6,52 medicamentos/gestante. Dentre esses, 85 $(14,96 \%)$ apresentam ação antimicrobiana, sendo que 194 $(34,1 \%)$ são de uso tópico, e 374 (65,9\%) de uso sistêmico. 
Dos 85 antimicrobianos prescritos, verificaram-se 13 produtos distintos. Os mais prescritos foram miconazol creme vaginal, cefalexina $500 \mathrm{mg}$, metronidazol creme vaginal, azitromicina $500 \mathrm{mg}$ e amoxicilina $500 \mathrm{mg}$.

Do total de mulheres que utilizaram medicamentos durante a gestação no período do estudo, 46 (52,9\%) receberam prescrições de antimicrobianos.

Ressalta-se que entre as 46 mulheres que receberam prescrição contendo antimicrobianos durante a gestação, observou-se a prescrição de um antimicrobiano para $24(54,4 \%)$ mulheres, dois para $10(21,7 \%)$, três para
$6(13,1 \%)$, quatro para $3(6,5 \%)$ e mais de cinco para 2 $(4,4 \%)$. Vale ressaltar que esses números não se referem a uso concomitante e sim durante todo o período de gestação.

A Tabela III apresenta o trimestre em que as gestantes estudadas receberam os antimicrobianos, podendo-se observar que os produtos ginecológicos foram os mais prescritos.

Quantos aos antimicrobianos prescritos às gestantes, agrupados conforme a categoria de risco estabelecida pela $F D A$, observou-se que oito $(61,54 \%)$ antimicrobianos da categoria B, quatro $(30,77 \%)$ da categoria $\mathrm{C}$.

Tabela II - Número e média de medicamentos utilizados pelas gestantes em relação ao número de consultas pré-natal atendidos na UBS Central e no CAAMI do município de Ijuí-RS, no ano de 2010. Ijuí/RS, 2011.

\begin{tabular}{lccc}
\hline Consultas & Gestantes n(\%) & Medicamentos n(\%) & Média de medicamento/gestantes \\
\hline$\geq 3$ & $19(21,84)$ & $106(18,66)$ & 5,58 \\
$4-6$ & $25(28,74)$ & $144(25,35)$ & 5,76 \\
$7-9$ & $29(33,33)$ & $206(36,27)$ & 7,10 \\
$\geq 10$ & $14(16,09)$ & $112(19,72)$ & 8 \\
Total & $87(100)$ & $658(100)$ & \\
\hline
\end{tabular}

Correlação de Spermann: $r=0,281 ; p=0,008$.

Tabela III - Distribuição das gestantes de acordo com o medicamento antimicrobiano prescrito e o período gestacional atendidas UBS Central e no CAAMI do município de Ijuí-RS, no ano de 2010. Ijuí/RS, 2011.

\begin{tabular}{lcccc}
\hline Antimicrobiano & $\begin{array}{c}\mathbf{1}^{\mathbf{0}} \text { trimestre } \\
(\mathbf{n} \%)\end{array}$ & $\begin{array}{c}\mathbf{2}^{\mathbf{0}} \text { trimestre } \\
\mathbf{( n \% )}\end{array}$ & $\begin{array}{c}\mathbf{3}^{\mathbf{0}} \text { trimestre } \\
(\mathbf{n} \%)\end{array}$ & $\begin{array}{c}\text { Total } \\
\text { produtos }\end{array}$ \\
\hline Miconazol CV* 2\% & $16(20)$ & $7(23,33)$ & $3(12)$ & $16(18,82)$ \\
Cefalexina 500 mg & $2(6,67)$ & $7(23,33)$ & $4(16)$ & $13(15,29)$ \\
Metronidazol CV & $5(16,67)$ & $4(13,33)$ & $3(12)$ & $12(14,12)$ \\
Azitromicina 500 mg & $2(6,67)$ & $4(13,33)$ & $5(20)$ & $11(12,94)$ \\
Amoxicilina 500 mg & $6(20)$ & $3(10,01)$ & $2(8)$ & $11(12,94)$ \\
Metronidazol 250 mg & $2(6,67)$ & $2(6,67)$ & $3(12)$ & $7(8,24)$ \\
Fluconazol 150 mg & $4(13,33)$ & $2(6,67)$ & - & $6(7,06)$ \\
Sulf. 400mg/trim.80 mg** & - & $1(3,33)$ & $3(12)$ & $4(4,71)$ \\
Outros & $3(10)$ & - & $2(8)$ & $5(5,88)$ \\
Total & $30(100)$ & $30(100)$ & $25(100)$ & $85(100)$ \\
\hline
\end{tabular}

$* \mathrm{CV}=$ creme vaginal; ** Sulfametoxazol e trimetroprima; ***Correlação de Spermann $\mathrm{r}=0,281 ; \mathrm{p}=0,008$.

\section{DISCUSSÃO}

Gestantes estão frequentemente expostas a medicamentos $^{(12)}$. Estudo realizado em São Paulo com 1.581 mulheres demonstrou que $97,6 \%$ delas utilizou pelo menos um medicamento durante a gestação e $2,2 \%$ fez uso de mais de dez, com média de 4,2 medicamentos por mulher ${ }^{(3)}$. De 100 mulheres que fizeram pré-natal em serviços do SUS em Santa Rosa/RS, 90\% utilizaram pelo menos um medicamento durante a gestação, totalizando 371 itens, sendo que a média foi de 4,1 medicamentos/gestante ${ }^{(12)}$. Um 
estudo avaliou a prescrição de medicamentos para gestantes em cinco UBSs do município de Ijuí/RS, e verificou que foram prescritos em média 3,5 medicamentos/gestante ${ }^{(13)}$, o que corresponde a metade da média de medicamentos prescritos no presente estudo (6,5 medicamentos/ gestante), indicando que nos locais estudados as gestantes apresentaram mais intercorrências no período gestacional.

Em estudo realizado em Ijuí/RS ${ }^{(13)}$ observou-se que grande parte das gestantes $(47,9 \%)$ realizou de 7 a 9 consultas de pré-natal, o mesmo número de consultas foi praticado por $33,33 \%$ das gestantes do presente estudo. Relacionou-se ${ }^{(13)} \mathrm{o}$ número de medicamentos prescritos com o número de consultas realizadas e constatou-se, da mesma forma que outra investigação ${ }^{(11)}$ realizada em Santa Rosa/ $\mathrm{RS}$, que o número de medicamentos prescritos aumenta com o número de consultas. Neste sentido, aparenta a sugestão ${ }^{(12)}$ de que a assistência pré-natal facilite o acesso das gestantes aos medicamentos. Com isso, apesar de ser aconselhado evitar, quando não há necessidade, o uso de fármacos nesse grupo populacional ${ }^{(4)}$, esse resultado reflete a utilização de medicamentos durante a gestação como uma prática frequente nas UBSs estudadas. Recomenda-se ${ }^{(13)}$ que antes de usar qualquer medicamento durante a gravidez, a gestante e o prescritor discutam a natureza deste, os efeitos que possa causar sobre o feto e se seu uso é absolutamente necessário. $\mathrm{O}$ ideal seria evitar o uso de qualquer tipo de medicamento durante a gravidez, o que raramente ocorre ${ }^{(14)}$.

Ainda com relação ao número de consultas de acompanhamento pré-natal, em Caxias do Sul/RS o número médio foi de $6,2^{(15)}$, o que se aproxima do valor encontrado no presente estudo, onde foram realizadas aproximadamente 5,3 consultas por gestantes. Em estudo ${ }^{(13)}$ realizado em Ijuí/RS, entre as cinco UBSs pesquisadas, destaca-se a Unidade Central, a qual também fez parte do presente estudo. Naquela investigação ${ }^{(13)}$, no ano de 2008 , a média de consultas realizadas foi 5,8 , sendo a média de medicamentos dispensados de 3,3 por gestante, números que se mantiveram durante o ano de 2010, conforme verificado no atual estudo.

As mulheres estão sendo estimuladas a realizar acompanhamento pré-natal e estão respondendo a esse chamado $^{(10)}$. O calendário de atendimento pré-natal deve ser programado em função dos períodos gestacionais que determinam maior risco materno e perinatal, sendo que devem ser realizadas no mínimo seis consultas, preferencialmente, uma no primeiro trimestre, duas no segundo e três no último trimestre. A maior frequência de visitas no final da gestação visa à avaliação do risco perinatal e das intercorrências clínico-obstétricas mais comuns nesse trimestre, como trabalho de parto prematuro, pré-eclâmpsia e eclâmpsia, amniorrexe prematura e óbito fetal ${ }^{(16)}$.
Neste sentido, as ações de saúde realizadas pelo Programa de acompanhamento pré-natal devem estar voltadas para a cobertura de toda a população-alvo da área de abrangência da unidade de saúde, assegurando continuidade no atendimento, acompanhamento e avaliação dessas ações sobre a saúde materna e perinatal. Ao Ministério da Saúde compete estabelecer políticas e normas técnicas para a atenção pré-natal de boa qualidade. Além dos equipamentos e instrumental necessários, deve-se levar em conta a capacitação da equipe de saúde ${ }^{(17)}$.

Por outro lado, evidencia-se que as consultas de prénatal realizadas têm facilitado o acesso das gestantes aos medicamentos. Estudo sobre a função social e psicológica da prescrição de medicamentos mostra que, para o médico, a prescrição não é apenas a documentação de sua intervenção, de modo a alterar a evolução de uma doença ou eliminar um sintoma, mas constitui um sinal de atenção com o paciente $^{(18)}$. Não receber uma prescrição de medicamentos pode ser interpretado como desinteresse e até mesmo incompetência do médico e representar a ruptura da relação do médico com sua clientela ${ }^{(18)}$.

Foram prescritos no presente estudo aproximadamente quatro vezes mais antibióticos de uso sistêmico do que em uma pesquisa $^{(18)}$ realizada em hospital-escola em São Paulo, que verificou que antibióticos sistêmicos foram usados por apenas $15,8 \%$ das pacientes.

Em um estudo ${ }^{(8)}$ realizado em serviços de pré-natal do SUS em seis cidades brasileiras, observou-se que $11,1 \%$ das gestantes receberam prescrição de antimicrobianos, correspondendo a $6,9 \%$ dos medicamentos utilizados por elas. Os grupos mais prescritos foram penicilinas $(40 \%)$, tetraciclinas $(30 \%)$ e sulfonamidas associadas à trimetoprima (11\%). Outra investigação ${ }^{(13)}$ verificou que $13,90 \%$ dos medicamentos prescritos para as gestantes eram anti-infecciosos de uso sistêmico, sendo este o terceiro grupo anatômico mais prescrito, com resultado semelhante encontrado em Santa $\operatorname{Rosa}^{(12)}$, onde os anti-infecciosos apresentaram 14,2\% dos medicamentos prescritos.

Estudo realizado para avaliar a qualidade do uso de antimicrobianos por gestantes de um hospital de Cuba entre 2007- 2009 evidenciou que a doença que mais motivou esse uso foi a sepse vaginal, diagnosticada de forma clínica em sua maioria e os antimicrobianos mais prescritos foram a azitromicina, o metronidazol, o clotrimazol e a nistatina ${ }^{(19)}$.

Os antimicrobianos de ação sistêmica mais frequentemente prescritos para as gestantes estudadas na presente pesquisa foram penicilinas e cefalosporinas, que, segundo um estudo ${ }^{(20)}$, atuam interferindo na síntese dos peptidoglicanos na parede celular bacteriana após ligação às proteínas de ligação de $\beta$-lactâmicos. E são antibióticos seguros e prescritos habitualmente para gestantes, incluindo 
cefalexina, ampicilina/amoxicilina e nitrofurantoína ${ }^{(20)}$. Os antibióticos que agem por inibição da síntese da parede celular apresentam baixo risco de efeitos colaterais, permitindo sua utilização com grande segurança em pediatria, na gravidez e lactação ${ }^{(21)}$. Os antimicrobianos supracitados encontram-se entre os medicamentos de escolha na atenção pré-natal, ao parto e ao puerpério ${ }^{(16)}$.

Considerando os antimicrobianos mais prescritos às gestantes estudadas na presente pesquisa, $30(35,3 \%)$ foram prescritos no primeiro trimestre de gestação, 30 $(35,3 \%)$ no segundo e $25(29,4 \%)$ no terceiro trimestre. Os riscos inerentes ao uso de fármacos durante a gravidez podem acontecer em qualquer fase, porém, os fármacos apresentam efeitos mais intensos quando administrados durante o primeiro trimestre ${ }^{(14)}$, principalmente entre a $3^{\mathrm{a}} \mathrm{e}$ a $11^{a}$ semana $^{(11)}$. Acredita-se que, no período de fertilização e de implantação (até 17 dias), os fármacos tóxicos possam interromper a gestação ou não causar efeito algum. No período de organogênese (de 18 a 56 dias), a suscetibilidade a malformações é máxima e no período fetal (a partir do $56^{\circ}$ dia), o risco de malformações morfológicas é baixo. Entretanto, as substâncias prejudiciais administradas nesse período podem causar alterações orgânicas funcionais e, também, comportamentais, sociais e intelectuais ${ }^{(4)}$.

Com o propósito de orientar o prescritor na escolha terapêutica mais adequada para uma gestante, a FDA classificou os fármacos quanto aos efeitos na gestação em categorias de risco $^{(11)}$. Conforme essa classificação, encontrou-se na presente investigação a prescrição às gestantes de oito $(61,54 \%)$ antimicrobianos da categoria B, quatro $(30,77 \%)$ da categoria $\mathrm{C}$ e nenhum das categorias $\mathrm{D}$ e X. Salienta-se que nenhum dos antimicrobianos foi classificado na categoria A. Tais resultados mostram que os antimicrobianos mais prescritos para as gestantes são os da categoria $\mathrm{B}$, estando de acordo com outros estudos ${ }^{(5 ; 13)}$.

Os medicamentos da categoria de risco $\mathrm{C}$ foram os segundos medicamentos mais prescritos em outro estudo ${ }^{(13)}$, o que também foi evidenciado na presente pesquisa, entretanto, a categoria de risco predominante em ambos foi a B.

Considerando a grande utilização de antimicrobianos da categoria de risco C, destaca-se a necessidade de revisar as prescrições emitidas para esse grupo, visto que nessa categoria estão medicamentos nos quais os estudos foram feitos em animais, que revelaram efeitos adversos sobre o feto ${ }^{(10)}$. É importante considerar que os dados obtidos a partir de estudos com animais não podem ser, integralmente, extrapolados para a gravidez humana ${ }^{(14)}$. Nessa categoria encontram-se antimicrobianos de uso sistêmico, como fluconazol, que foi prescrito a cinco $(5,75 \%)$ gestantes da atual pesquisa. Este fármaco apresenta ação antifúngica, e reações adversas pouco frequentes, que incluem distúrbios gastrointestinais, cefaléia, ou, mais raramente, hepatotoxicidade e trombocitopenia ${ }^{(22)}$, ou mesmo, aborto, prolongamento do parto e anormalidades fetais em estudos realizados com fluconazol em animais ${ }^{(22)}$.

Miconazol de uso ginecológico, medicamento mais prescrito no presente estudo, é um derivado azólico de amplo espectro de ação ${ }^{(22)}$, que atua inibindo a síntese do ergosterol, componente essencial da membrana celular fúngica ${ }^{(4)}$. É amebicida tecidual e tem sido a primeira escolha para casos de infecção ativa por Entamoeba histolytica, com eficácia estimada em $90 \%{ }^{(21)}$. Seu emprego sistêmico foi praticamente abandonado devido à toxicidade ${ }^{(4)}$. O miconazol, nas suas variadas formas farmacêuticas, é o único antifúngico que consta na relação de medicamentos utilizados no pré-natal, parto e puerpério, provavelmente devido ao seu pequeno efeito sistêmico (vaginal 1,4\% e dérmica menor que $0,013 \%)^{(15)}$, o que o torna menos tóxico.

A seleção de um antibiótico para uso tópico fundamenta-se principalmente no seu espectro de ação, na etiologia do processo infeccioso e na possibilidade de determinar reações irritantes primárias ou de sensibilização. Contudo, é importante salientar que a antibioticoterapia tópica apresenta certas desvantagens que limitam suas indicações, entre as quais citam-se o tempo transcorrido até o efeito farmacológico e os fatores que influenciam a absorção, como a concentração e a natureza do princípio ativo, o estado da pele e o comportamento do veículo ${ }^{(22)}$

Do total de gestantes investigadas no atual estudo, $6,89 \%$ receberam prescrições de antimicrobianos pertencentes à categoria de risco $\mathrm{C}$ durante o primeiro trimestre da gestação. Os medicamentos dessa categoria devem ser evitados no primeiro trimestre, devido aos riscos de anormalidades congênitas ${ }^{(10)}$. Com isso, sugere-se que as gestantes que impreterivelmente necessitarem fazer uso de fármacos pertencentes a essa categoria de risco durante a gestação sejam acompanhadas durante o período de utilização e os resultados obtidos sejam registrados para que possam ser socializados e comparados, a fim de beneficiar outras mulheres que possam vir se encontrar na mesma situação ${ }^{(23)}$.

Verificando os antimicrobianos da categoria B utilizados na presente pesquisa, destacam-se cefalexina, azitromicina e amoxicilina. A indicação destes medicamentos se justifica por serem antimicrobianos que podem ser indicados para uso na gestação por apresentarem alta toxicidade seletiva e pequeno potencial de toxicidade para a gestante e feto $^{(7)}$. Cefalexina foi o antibiótico de uso sistêmico mais prescrito $(14,12 \%)$ para as gestantes estudadas na pesquisa em questão. Outro estudo ${ }^{(12)}$ refere-se à cefalexina como o quarto medicamento mais prescrito $(19,3 \%)$ em estudo realizado no mesmo município, também com gestantes atendidas no serviço público de saúde. 
Nitrofurantoína pertence à mesma categoria de risco, podendo causar hemólise neonatal a partir do terceiro trimestre $^{(10)}$. No presente estudo, esse medicamento foi prescrito a uma mulher no segundo trimestre da gestação.

Quanto aos antimicrobianos pertencentes às categorias de risco $\mathrm{D}$ e X, não se encontrou registros de dispensação na investigação em tela, o que pode ser justificado pelo baixo número de antimicrobianos pertencentes a essas classes. A ausência de medicamentos dessas categorias possivelmente está relacionada com o fato de os medicamentos que são padronizados pelo Ministério da Saúde para esse grupo (gestantes), não pertencerem a essas categorias ${ }^{(16)}$.

Durante a gestação, a ITU é a infecção mais frequente, estimando-se que até $10 \%$ das gestantes serão acometidas por algum episódio de infecção urinária, sendo a maioria assintomática ${ }^{(4)}$. Os micro-organismos envolvidos em cerca de 80 a $90 \%$ dessas infecções são os da flora perineal normal. Os gram-negativos, como Klebsiela principalmente a Escherichia coli, Enterobacter e Proteus, respondem pela maioria dos outros casos, além do enterococo e do streptococo do grupo B. A bacteriúria assintomática é mais frequente, sendo que as infecções sintomáticas acometem o trato urinário inferior (cistites), ou ainda, o trato superior (pielonefrite) $^{(16)}$, sendo também complicações comuns a esse grupo ${ }^{(4)}$.

Uma pesquisa ${ }^{(13)}$ também não encontrou medicamentos da categoria de risco $X$ prescritos a gestantes em outras UBSs de Ijuí/RS. Já uma investigação ${ }^{(17)}$ que avaliou medicamentos prescritos às gestantes e a automedicação realizada por elas, constatou a utilização de um medicamento desta categoria, comprovadamente teratogênico (misoprostol), o qual tinha sido utilizado na tentativa de provocar aborto. Porém, outra pesquisa $^{(8)}$ na qual foram verificados os riscos ao feto através de prescrições de gestantes em São Paulo, foi notável o elevado índice de medicamentos da categoria $\mathrm{X}$ $(3,5 \%)$.

Considerando as categorias de risco em que os medicamentos prescritos estão inseridos e o trimestre de gestação, verificaram-se na presente pesquisa prescrições contendo medicamentos de risco B em todos os períodos da gestação. Nessa categoria estão inseridos medicamentos em que estudos em reprodução animal não demonstraram risco fetal; ou os estudos em reprodução animal demonstraram efeito adverso que não foi confirmado em estudos controlados em mulheres no primeiro trimestre de gravidez (e não há evidência de risco nos demais trimestres), sendo necessária certa cautela com relação à prescrição destes medicamentos $^{(11)}$. No presente estudo, dos antimicrobianos classificados na categoria $\mathrm{C}$, apenas um medicamento de uso ginecológico foi prescrito nos três trimestres, os demais foram prescritos apenas no primeiro e segundo trimestres, o que não está em conformidade com a literatura ${ }^{(10)}$, pois alguns dos medicamentos pertencentes a essa categoria de risco devem ser evitados no primeiro trimestre de gestação pelo risco de causar anormalidades congênitas.

No presente estudo, e em outros semelhantes realizados no Brasil ${ }^{(3,5,13,15)}$, a maioria das gestantes encontrava-se na faixa etária de 20 a 29 anos. Porém, segundo estudo realizado no ano de 2008 em Caxias do Sul/RS, as taxas de gravidez na adolescência ainda continuam elevadas, sendo $26,2 \%$ menores de 20 anos $^{(15)}$. Outro estudo ${ }^{(13)}$ realizado também em UBSs de Ijuí/RS encontrou apenas seis (3,5\%) gestantes apresentando idade entre 14 e 19 anos, já no atual estudo, foram encontradas $12(13,79 \%)$ nessa faixa etária.

Não existem dúvidas de que gestantes estão frequentemente expostas a medicamentos em função de intercorrências características desse período. As infecções representam uma relativa fonte de complicações maternas, o que reflete na prescrição e uso de antimicrobianos. $\mathrm{O}$ acesso à assistência pré-natal e a antibióticos por essas gestantes não implica necessariamente em melhores condições de saúde ou qualidade de vida, pois os maus hábitos prescritivos, muitas vezes sem necessidade, ou até mesmo a falta de informações sobre alguns antibióticos prescritos podem levar a tratamentos pouco seguros, tanto para as gestantes quanto para o feto ${ }^{(24)}$.

Os resultados apresentados mostram a necessidade de ações que visem à promoção de conhecimentos relacionados ao uso de medicamentos no período gestacional, visto que é de suma importância que a equipe de profissionais de saúde tenha conhecimento dos medicamentos que podem ser utilizados para esse grupo, sem apresentar danos ou riscos. O planejamento de intervenções educativas para gestantes, desestimulando a prática da automedicação, e atividades de atualizações para profissionais da saúde parecem imprescindíveis para uma gestação com qualidade e para minimizar riscos ao feto. É importante destacar que não houve contato com as gestantes, limitando o acesso a algumas informações, como a utilização de medicamentos prescritos que tenham sido adquiridos em outros locais que não na farmácia da rede municipal de saúde, bem como a utilização de medicamentos sem prescrição médica, ou ainda o uso de plantas medicinais, tratando-se esta de uma limitação do estudo. Neste sentido, destaca-se que cada uma destas práticas expõe ainda mais a gestante e o feto a riscos inerentes ao efeito individual dos medicamentos utilizados, bem como a interações entre eles.

\section{CONCLUSÃO}

Identificaram-se medicamentos antimicrobianos entre os prescritos a $52,9 \%$ das gestantes atendidas no Programa Nacional de Acompanhamento Pré-Natal e Puerpério em um município do Rio Grande do Sul no ano de 2010. Os 
produtos prescritos, na sua maioria, são de uso sistêmico, miconazol creme vaginal, cefalexina $500 \mathrm{mg}$, metronidazol creme vaginal, azitromicina $500 \mathrm{mg}$ e amoxicilina $500 \mathrm{mg}$ foram os mais prescritos. A maioria dos produtos apresenta risco $\mathrm{B}$ na gestação, o que de acordo com o $F D A$ significa que não há evidência direta de risco ao concepto, mas que estudos em animais mostraram algum risco fetal.

\section{AGRADECIMENTOS}

Aos funcionários da UBS Central e do CAAMI.

\section{REFERÊNCIAS}

1. Carmo TA. Medicamentos e gravidez. Saúde Rev. 2003;5(10):55- 61 .

2. Brum LFS, Pereira P, Felicetti LL, Silveira RD. Utilização de medicamentos por gestantes usuárias do Sistema Único de Saúde no município de Santa Rosa (RS, Brasil). Ciênc Saúde Coletiva. 2011;16(5):243542.

3. Henderson E, Mackillop L. Prescribing in pregnancy and during breast feeding: using principles in clinical practice. Postgrad Med J. 2011;87(1027):349-54.

4. Wannmacher L. Fármacos em gestação e lactação. In: Fuchs FD, Wannmacher L. Farmacologia clínica. $3^{\mathrm{a}}$ ed. Rio de Janeiro: Guanabara Koogan; 2010. p. 1146-59.

5. Oliveira Filho AD, Gama DP, Leopardi MG, Dias JMG, Lyra JDP, Neves SJ. Aderência autorreferida a medicamentos prescritos durante a gestação. Rev Bras Ginecol Obstet. 2012;34(4):147-52.

6. Araújo DD, Leal MM, Santos EJV, Leal LB. Consumption of medicines in high-risk pregnancy: evaluation of determinants related to the use of prescription drugs and self-medication. Braz J Pharm Sci. 2013;49(3):491-9.

7. Furini AAC, Gomes AM, Silva CO, Vieira JKG, Silva VP, Atique, TSC. Estudo de indicadores de prescrição, interações medicamentosas e classificação de risco ao feto em prescrições de gestantes da cidade de Mirassol - São Paulo. Rev Ciênc Farm Básica Apl. 2009;30(2):211-16.

8. Mengue SS, Schenkel EP, Shmidt MI, Duncan BB. Fatores associados ao uso de medicamentos durante a gestação em seis cidades brasileiras. Cad Saúde Pública 2004;20(6):1602-8

9. Duarte G, Marcolin AC, Gonçalves CV, Quintana SM, Berezowski AT, Nogueira AA, et al. Infecção urinária na gravidez: análise dos métodos para diagnóstico e do tratamento. Rev Bras Ginecol Obstet. 2002;24(7):4717.

10. Silva EV, Hoefler R, Heineck I, Lopes LC, Falkenberg MB, Lisboa SSML, et al. Fármacos e Gravidez. In: Ministério da Saúde (BR), Secretaria de Ciência, Tecnologia e Insumos Estratégicos, Departamento de Assistência Farmacêutica e Insumos Estratégicos. Formulário terapêutico nacional: Rename 2010. Brasília: Ministério da Saúde; 2010. p. 1071-85.

11. Meadows M. Pregnancy and the drug dilemma. FDA Consumer [internet] 2001 [acesso em 2013 Jan 16]. Disponível em: http://www.fda.gov.fdac/ feature/2001/301-preg.html

12. Brum LFS, Pereira P, Felicetti LL, Silveira RD. Utilização de medicamentos por gestantes usuárias do Sistema Único de Saúde no município de Santa Rosa/ RS. Ciênc Saúde Coletiva. 2011;16(5):2435-42.

13. Buzetto T, Bueno CS, Oliveira KR. Prescrição de medicamentos para gestantes atendidas pelo Programa de Acompanhamento Pré-natal do município de IjuíRS, Brasil. Latin Am J Pharm. 2011;30(1):81-8.

14. Monteiro MP. Informações para o uso de medicamentos na gravidez e lactação. Fortaleza: Universidade Federal do Ceará; 2008.

15. Trevisan MR, Lorenzi DR, Araújo NM, Ésber K. Perfil da assistência pré-natal entre usuárias do Sistema Único de Saúde em Caxias do Sul. Rev Bras Ginecol Obstet. 2002;24(5):293-9.

16. Ministério da Saúde (BR), Secretaria de Atenção à Saúde, Departamento de Ações Programáticas Estratégicas. Pré-natal e puerpério: atenção qualificada e humanizada: manual técnico. Brasília: Ministério da Saúde; 2005.

17. Ministério da Saúde (BR), Secretaria de Políticas de Saúde. Assistência pré-natal: manual técnico. Brasília: Ministério da Saúde; 2000.

18. Fonseca MRCC, Fonseca E, Bergsten-mendes G. Prevalência do uso de medicamentos na gravidez: uma abordagem farmacoepidemiológica. Rev Saúde Pública. 2002;36(2):205-12.

19. Pavón PJ, Diaz MM, Mateu LL, Rojas GJ. Utilización de antimicrobianos en el Hogar materno Doña Leonor Pérez durante el período 2007-2009. Rev Cuba Farm. 2013;47(1):86-98.

20. Rang HP, Dale MM, Ritter JM. Farmacologia. 7a ed. Rio de Janeiro: Guanabara Koogan; 2012. Cap. 43; p. 576-93. 
21. Fiol FSD, Toledo MI. Cefalosporinas. In: Ministério da Saúde (BR), Secretaria de Ciência, Tecnologia e Insumos Estratégicos. Departamento de Assistência Farmacêutica e Insumos Estratégicos. Formulário terapêutico nacional 2010: Rename 2010. Brasília: Ministério da Saúde; 2010. p. 117-9.

22. Machado A, Barros E. Antibacterianos. In: Barros E, Barros HMT. Medicamentos na prática clínica. Porto Alegre: Artmed; 2010. p. 396-478.

23. Farina SS, Fiol FSD. Imidazólicos. In: Ministério da Saúde (BR), Secretaria de Ciência, Tecnologia e Insumos Estratégicos, Departamento de Assistência Farmacêutica e Insumos Estratégicos. Formulário terapêutico nacional 2010: Rename 2010. Brasília: Ministério da Saúde; 2010. p. 126-7.

24. Arrais PSD, Barreto ML, Coelho HLL. Aspectos dos processos de prescrição e dispensação de medicamentos na percepção do paciente: estudo de base populacional em Fortaleza, Ceará, Brasil. Cad Saúde Pública 2007; 23(4):927-37.

\section{Endereço do primeiro autor:}

Janaína Soder Fritzen

Universidade Regional do Noroeste do Estado do Rio

Grande do Sul

Departamento de Ciências da Vida

Rua do Comércio, 3000

Bairro: Universitário

CEP: 98700-000 - Ijuí - RS - Brasil

E-mail: janaina.fritzen@unijui.edu.br

\section{Endereço para correspondência:}

Christiane Fátima Colet

Universidade Regional do Noroeste do Estado do Rio Grande do Sul

Departamento de Ciências da Vida

Rua do Comércio, 3000

Bairro: Universitário

CEP: 98700-000 - Ijuí - RS - Brasil

E-mail: christiane.colet@unijui.edu.br 